\title{
Study of the mechanism of protonated histidine-induced conformational changes in the Zika virus dimeric envelope protein using accelerated molecular dynamic simulations
}

\author{
Jixue Sun, ${ }^{a}$ Yang Li, ${ }^{a}$ Pi Liu, ${ }^{* b}$ Jianping $\operatorname{Lin}^{{ }^{*}, \mathrm{~b}}$
}

a State Key Laboratory of Medicinal Chemical Biology, College of Pharmacy and Tianjin Key Laboratory of Molecular Drug Research, Nankai University, Haihe Education Park, 38 Tongyan Road, Tianjin 300353, People’s Republic of China.

b Tianjin Institute of Industrial Biotechnology, Chinese Academy of Sciences, 32 West 7th Avenue, Tianjin Airport Economic Area, Tianjin 300308, People’s Republic of China.

\section{Correspondence}

Jianping Lin, State Key Laboratory of Medicinal Chemical Biology, College of Pharmacy and Tianjin Key Laboratory of Molecular Drug Research, Nankai University, Haihe Education Park, 38 Tongyan Road, Tianjin 300353, People’s Republic of China. Emails: jianpinglin@nankai.edu.cn;

Pi Liu, Tianjin Institute of Industrial Biotechnology, Chinese Academy of Sciences, 32 West 7th Avenue, Tianjin Airport Economic Area, Tianjin 300308, People’s Republic of China. Email: liu_p@tib.cas.cn. 


\begin{abstract}
The Zika virus has drawn worldwide attention because of the epidemic diseases it causes. It is a flavivirus that has an icosahedral protein shell constituted by an envelope glycoprotein (E-protein) and membrane protein (M-protein) in the mature virion. The multistep process of membrane fusion to infect the host cell is $\mathrm{pH}$-induced. To understand the mechanism of the conformational changes in the $(\mathrm{E}-\mathrm{M})_{2}$ protein homodimer embedded in the membrane, two 200-ns accelerated dynamic simulations were performed under different $\mathrm{pH}$ conditions. The low $\mathrm{pH}$ condition weakens the interactions and correlations in both E-protein monomers and in the E-M heterodimer. The highly conserved residues, His249, His288, His323 and His446, are protonated under low $\mathrm{pH}$ conditions and play key roles in driving the fusion process. The analysis and discussion in this study may provide some insight into the molecular mechanism of Zika virus infection.
\end{abstract}




\section{Introduction}

The Zika virus (ZIKV), a mosquito-borne arbovirus transmitted to humans through a mosquito (Aedes aegypti) vector[1,2], has caused a recent outbreak of an autoimmune neurological syndrome and microcephaly in foetuses when pregnant women were infected[3-5]. Currently, the spread of ZIKV is a focus of scientists and researchers worldwide because of its high pace and wider geographic range[6-8].

$\mathrm{ZIKV}$ is a member of the flavivirus genus.Flaviviruses are a group of enveloped positive-stranded RNA viruses including approximately 70 pathogens, such as the yellow fever virus, dengue virus (DENV), West Nile virus (WNV), tick-borne encephalitis virus (TBEV) and Japanese encephalitis virus (JEV)[9,10]. Flaviviruses share a conserved sequence and similar architecture[11,12]. The virion of the flavivirus has an icosahedral protein shell that is composed of 180 identical capsid proteins that are composed of an envelope glycoprotein (E-protein) and precursor membrane protein (prM-protein) or membrane protein (M-protein)[13-15] and are positioned in a lipid membrane derived from its host cell[16,17]. Membrane fusion of the flavivirus is mediated by the E-protein, a class II viral fusion protein that has three domains (DI, DII and DIII) and transmembrane (TM) and stem regions (Fig. 1)[18-21]. The hydrophobic fusion loop (FL) at the end of DII is crucial to insert the membrane into the host cell[22,23].

Some work has confirmed that both the multistep process of virus maturation and membrane fusion are triggered in a low-pH environment[24-26]. When the pH drops,the 180 identical E-prM heterodimers(in the form of 60 trimers) form 90 (E-prM $)_{2}$ homodimers. Then the prM-protein decomposes to the M-proteinto expose the FL buried in the hydrophobic pocket[27,28]. This is followed by 90 $(\mathrm{E}-\mathrm{M})_{2}$ homodimer dissociation and conversion to 60 trimers to prepare for 
membrane fusion[20,29]. This $\mathrm{pH}$-introduced fusion event is thought to be an appropriate target for interfering with the virus infection.

Over the last decade, $\mathrm{pH}$-introduced conformational changes in flaviviruses, especially DENV, were extensively explored using experimental and computational methods[30-37]. The X-ray crystal structures of TBEV, WNV, JEV and DENV confirmed that the mature E protein can adopt different conformations under neutral and low pH conditions[16,26,38-42]. For DENV, theicosahedral shell has a spiky surface at $\mathrm{pH}=8.0$ with 60 protrusions, whereas it has a smooth surface at $\mathrm{pH}=6.0[16,26]$, which is mainly involved in the conformational transformation of the de-polymerization of $(\mathrm{E}-\mathrm{M})_{2}$ homodimers and re-association of $(\mathrm{E}-\mathrm{M})_{2}$ homodimers to expose the FL[35].Meanwhile, the protonation of a small number of conserved histidine residues is closely related to the fusion event in the envelop protein of both DENV and TBEV, were known as the histidine switch hypothesis[43]. In the DENV E protein, His144 and His317 (corresponding to His146 and His323 in TBEV) have been proposed to break the contacts of DI/DIII, whichhas contributed to initial stages of membrane fusion[38]. As for TBEV, the conserved histidine residues were explored by mutational analysis, His323had been pointed as one of the $\mathrm{pH}$ sensors for the process of membrane fusion and directly involved in other $\mathrm{pH}$-dependent process in the work of Fritz et al.[44].Whereas,there are few atomic details to depict membrane fusion of flavivirus, even for $(\mathrm{E}-\mathrm{M})_{2}$ proteins.

Recently, the structure of the mature ZIKV strain H/PF/2013 from cryo-electron microscopy was reported by Lok’s groupand Kuhn's group $[45,46]$. This was the first time that the ZIKV whole envelope protein was solved in a mildly high $\mathrm{pH}$ environment (at a pH of 8.0 in the work of Lok's group). These overall structures make it possible to understand the molecular mechanism of the infection and to design appropriate structural-based drugs and vaccines for ZIKV. 
The entire membrane fusion process occurs within a few seconds[24], which is insufficient for a cMD study on the nanosecond timescale to simulate large conformational changes[47]. The enhanced sampling technique, accelerated dynamic simulation (aMD), can capture the millisecond timescale within hundreds of nanoseconds[48]. To explore the conformational changes under low $\mathrm{pH}$ conditions and to investigate the $\mathrm{pH}$-introduced membrane fusion mechanism, we performed two 200-ns aMD simulations forZIKV (E-M $)_{2}$ embedded in a simplified membrane using both single and double protonation of histidines. The potential of the mean force calculation (PMF) and dynamic cross-correlation map (DCCM) were used to study the conformational changes, and they indicated that low $\mathrm{pH}$ weakens the interactions and correlations in both E-protein monomers (monomer 1 and monomer 2) and the E-M heterodimer. The key roles for driving the fusion process of residues His249, His288, His323 and His446, which are highly conserved in the E proteins of all flaviviruses, were explored. This analysis and discussion may provide some insight into the molecular mechanism of ZIKV infection.

\section{Materials and Methods}

\section{Systems}

The previously reported X-ray structure solved by Lok et al. (PDB ID: 5IZ7)[45]was used as the starting structure, and chains A, C, D and F were maintained to obtain the $(\mathrm{E}-\mathrm{M})_{2}$ homodimers. Using the histidine switch hypothesis mentioned above, two systems were utilized to emulate the effects of low pH (low_pH) and neutral pH (neutral_pH) by modifying the protonated states of the histidine residues. All histidine residues were double protonated in the low_pH system[31,32,49]. The protonation states of other titratable groups were determined at pH6.0 and 7.0 for the low_pH and neutral_pH systems, 
respectively, using a $\mathrm{H}++$ program[50]. All of the disulphide bonds found in the crystal structure were preserved.

Each system was inserted into a $180 \times 90 \quad \AA^{2}$ palmitoyl-oleoyl-phosphatidyl-choline (POPC) lipid bilayer using the membrane plugin in VMD[51]. The membrane was parallel to the xy-plane. The $(\mathrm{E}-\mathrm{M})_{2}$ homodimers was solvated in a box using TIP3P water[52] and neutralized using $0.15 \mathrm{M} \mathrm{NaCl}$. Hydrogen atoms were added using the Leap module of the Amber14 package[53]. The lipid/water molecules that were located within $3 \AA$ of the protein were removed. Each of the systems included approximately 173,500 atoms. The final dimension of the water-lipid box was $180 \times 90 \times 115 \AA^{3}$, and the size of protein was $150 \times 56 \times 71 \AA^{3}$ (Fig. S1)

\section{Molecular dynamic simulations}

In the MD simulations of the low_pH system and neutral_pH system, an amber FF14SB force field[54] was used for protein, and amber lipid force fields, LIPID14[55]and GLYCAM06[56], were used for the POPC and glycan, respectively. The glycan at the ASN154 of each envelope protein was in the MD simulations but it was not part of the studied subject. The 10,000 step minimization was performed for both systems (5,000 steps for the steepest descent[57] minimization and 5,000 steps for the conjugate gradient[58] minimization), followed by a 500-ps constant volume (NVT) MD simulation to heat the systems from 0 to $310 \mathrm{~K}$. A force constant of $20 \mathrm{kcal} \cdot \mathrm{mol}^{-1} \cdot \AA^{-2}$ was used to constrain without the tail of POPC during heating. A Langevin thermostat[59] was used for temperature control. To equilibrate the membrane, a 20-ns $\mathrm{MD}$ at a constant pressure (NPT, $\mathrm{P}=1 \mathrm{~atm}$ ) and at $310 \mathrm{~K}$ was performed with a weak force constant $\left(20 \mathrm{kcal} \cdot \mathrm{mol}^{-1} \cdot \AA^{-2}\right.$ for the first $10 \mathrm{~ns}$ and $10 \mathrm{kcal} \cdot \mathrm{mol}^{-1} \cdot \AA^{-2}$ for the remaining time) while a harmonic constraint was applied to protein. Then, a 20-ns cMD simulation for each system was performed without any constraints. The cut-off value of the Van der Waals interactions was set to $10 \AA \AA$. The Particle Mesh Ewald (PME) method[60] was 
used to calculate the long-range electrostatic interactions. The van der Waals interactions were modelled by the Lennard-Jones potential. The SHAKE algorithm[61] was used to restrain all of the bond lengths that involved hydrogen atoms.

After the cMD simulations,aMD simulations were performed on the two systems to determine the long-lasting conformational transitions. In the aMD protocol, a "boost potential", $\Delta V(r)$, was added to the original dihedral potential, $V(r)$. The energy, $V^{*}(r)$, can be expressed as follows[48]:

$$
\begin{gathered}
V^{*}(r)=V(r)+\Delta V(r) \\
\Delta V(r)=\left\{\begin{array}{c}
0 \quad, V(r) \geq E \\
\frac{(E-V(r))^{2}}{\alpha+E-V(r)}, V(r)<E
\end{array}\right.
\end{gathered}
$$

where $E$ is the threshold energy and $\alpha$ is the acceleration parameter. With the aMD implemented in the AMBER MD package[53], we boosted the torsional terms of the potential in aMD. The parameters that must be specified in the aMD simulation are $E($ dih $)$ (average dihedral energy threshold) and $\alpha$ (dih) (inverse strength boost factor for the dihedral energy).

The average dihedral energy $\left(V_{\text {avg }}\right)$ in the cMD were used as references to compute $E($ dih $)$ and $\alpha(d i h)[62]$ :

$$
E(d i h)=V_{a v g}+V_{a v g} \times 0.3, \alpha(d i h)=V_{a v g} \times 0.3 / 5
$$

In both the low_pH and neutral_pH systems, 200-ns aMD simulations were performed after cMD by restarting from the final structure of the cMD simulation. Thus, a 220-ns MD simulation was performed for each system in total. The 2-fs step was used in the entire simulation, and snapshots of the system were saved every 10 ps.

\section{Analysis of the simulations}

CPPTRAJ and MMPBSA methods. The CPPTRAJ module[63] of AmberTools15 was used to calculate the root mean square deviations (RMSD), 
number of hydrogen bonds (H-bonds) and solvent accessible surface area (SASA) of each system.

The binding free energy was calculated using the MMPBSA method[64-66]. In total, 200 snapshots from the last 20-ns trajectory of each system were extracted. All of the parameters used during the calculation were set as default values. Since we were mainly interested in the differences between the binding free energies that we calculated, entropy was ignored in our calculations.

Potential of mean force (PMF). The potential of mean force (PMF)[67] was calculated to study the energy changes along with the conformational change of the $(\mathrm{E}-\mathrm{M})_{2}$ homodimer based on an adequate sampling using the whole MD productions. The energy landscape was calculated by[67]:

$$
\Delta G(x, y)=k_{B} T \ln g(x, y)
$$

where $k_{\mathrm{B}}$ is the Boltzmann constant, $T$ is the simulation temperature, and $g(x, y)$ is the normalized joint probability distribution. The minimum energy was set to be zero.

Dynamic cross-correlations map (DCCM). The dynamic cross-correlations map (DCCM)[68] of the residues in the $(\mathrm{E}-\mathrm{M})_{2}$ homodimer of the last 20 ns of the two trajectories were calculated based on mutual information between all $\mathrm{C}_{\alpha}$ atoms except for the glycosylation sites, Asn154 and Asn659 (residue numbers are 505 and 1010 in our simulation, respectively), in each monomer using the NetworkView plugin in VMD[51] and carma[69].

\section{Results and Discussion}

We performed two 220-ns MD simulations to observe the conformational changes induced by lowpH [24].The proteins were embedded into a simplified membrane during the MD simulations. So the effect of the bilayer in the model should be analyzed. In both the low_pH and neutral_pH system, the thickness of the bilayer proximal to the TM domains of the proteins decreases, leading to 
a curvature from the initial plane (Figs. 2A and 2B). This indicates that the curvature is irrelevant to the protonation of histidine, which has been observed experimentally[70]. The cryo-EM maps show that the bilayer lipid membrane of the ZIKV-antibody complex structure is located at similar radii at $\mathrm{pH} 8.0$ and pH 5.0. The thickness of bilayer was measured referring Sansom's work, which shows the similar curvature of the bilayer in the Dengue virus[71]. The lipids were categorized as TM domain-local or -distalusing a 6- $\AA$ distance threshold. The differences between $\mathrm{z}$ coordinates of the lipid head groups of each leaflet were calculated to estimate bilayer thickness. Figures 2C and 2D show time evolutions of the average thickness of bilayer of each system. The bilayer thickness distal from and local to the TM domains was $\sim 33$ and $\sim 28 \AA$, respectively. The degree of bilayer thinning was $5 \AA$. This agrees well with the simulation of a single $(\mathrm{E}-\mathrm{M})_{3}$ asymmetric unit of Dengue virus in a PC bilayer.

\section{Conformational change of the (E-M) $)_{2}$ homodimers}

The RMSDs of both the trajectories were calculated (Fig. S2). The neutral_pH system became steady at approximately $3.5 \AA$ after 80 ns, whereas the low_pH system fluctuated at approximately $4.5 \AA$. The low_pH system had a larger RMSD (almost 1-2 Å) than the neutral_pH system over time, indicating that the $(\mathrm{E}-\mathrm{M})_{2}$ homodimer may have some conformational changes at low $\mathrm{pH}$.

As mentioned above, the E proteins of ZIKV are class II fusion proteins. They undergo a rearrangement from a $(\mathrm{E}-\mathrm{M})_{2}$ homodimers to a trimer, which is induced by the low $\mathrm{pH}$ [19]. The rearrangement may make the integration uncompact when the process of membrane fusion is activated. Thus, two distances, D1 and D2, were measured in the directions parallel to the membrane and vertical to the membrane, respectively, to study the distance changes of the two systems (Fig. 3A). D1 is the distance between the centre of mass (COM) of the DII of each monomer, which mainly represented the geometrical change of the $(\mathrm{E}-\mathrm{M})_{2}$ homodimers in the $\mathrm{y}$-axis direction. D2 is the distance between the COM of DI, DII and DIII and COM of the TM region of the $(E-M)_{2}$ 
homodimers, which mainly represented the geometrical change in the z-axis direction. We calculated the PMF for the distance D1 vs. D2 (Fig. 3B). The PMF map clearly depicts the conformational state of each system: the low_pH state and neutral_pH state. Figures 3C and 3D show the time evolutions of the distance changes of D1 and D2 for each system (Fig. S3 shows scatter plots of D1 vs. D2 for each system). In the neutral_pH system, both distances D1 and D2 had no shifts from their initial distances and stabilized at $27.5 \AA$ and $24 \AA$, respectively. However, in the low_pH system, D1 and D2 showed obvious ascents to $29.5 \AA$ and $27.5 \AA$ over time, respectively.

The results indicates that the low_pH state underwent a larger conformational change than the neutral_pH state. The increased distances of D1 and D2 indicate that DII departs from another DII and that the E proteins move upwards from the membrane, respectively. The conformational changes are fundamental prerequisites for the rearrangement[20].

\section{Correlated motions induced by low pH}

To gain insight into the conformational changes of the dimer at low $\mathrm{pH}$, the DCCMs of the low_pH and neutral_pH system were calculated (Fig. 4A). Different colours were used to show the correlation coefficients of the different regions of the $(\mathrm{E}-\mathrm{M})_{2}$ homodimers. Obviously, in the low_pH system, the residue motions exhibited a reduction in the residue-residue correlations compared to the neutral_pH system. Three representative sites followed this reduction in the low_pH system. The residue-residue correlation coefficients of (a) residues 196-285 (DII of monomer 1) and residues 701-790 (DII of monomer 2), (b) residues 150-195 (DI of monomer 1) and residues 403-459 (the stem region of monomer 1), and (c) residues 98-109 (the FL of monomer 1) and residues 302-402 (the DIII of monomer 1) are lower than the correlation coefficients at the same sites in the neutral_pH system, indicating that the low $\mathrm{pH}$ weakens the correlations of these regions. 
The weakened interactions and conformational changes can be observed from the illustration of the alignment of the initial, low_pH and neutral_pH states based on the DI and DIII (Fig. 4B). To choose the representative low_pH and neutral_pH state,the200-ns aMD trajectory (2000 frames in total) of each system was clusteredbased on the distance D1 and D2. The calculated data of cluster were presented in Table S1. In each system, the cluster 1 is highly populated (70.2\% and $67.7 \%$, respectively) and the cluster 3 is probably insignificant. The representative low_pH and neutral_pH state was chosen from the centroid of the cluster 1 of each system, respectively. In the low_pH state, the DII moved upwards away from the stem and TM region, which corresponds with its biological function in the process of membrane fusion[23]. The COM of the FL increases by $17 \AA$ from the initial position. However, the DII in the neutral_pH state did not shift considerable compared with the low_pH state. This induced the reduced residue-residue correlation coefficients in the low_pH system (the blue frame in Fig. 4A).

\section{Energetic analysis}

Three binding free energies between (1) the E dimer proteins (E-E), (2) E proteins and $M$ proteins (E-M) and (3) E proteins and bilayer (E-P) were calculated to detect the inner interaction in terms of energy (Table 1). In the neutral_pH system, the binding free energies were $-103.19,-344.21$ and $-292.04 \mathrm{kcal} / \mathrm{mol}$, respectively, whereas in the low_pH system, the binding free energies increased to $-43.97,-246.00$ and $-229.25 \mathrm{kcal} / \mathrm{mol}$, respectively. The lower binding free energies indicate that the entire heterodimer is a compact integration. However, protonated residue motions lead to the reduced residue-residue correlation and the increased binding free energies between the $\mathrm{E}$ protein and the other $\mathrm{E}$ protein, the $\mathrm{M}$ protein and the bilayer.

The computed increased energy corresponds to the other flavivirus, DENV[32]. In Dubey's work, only E dimer proteins were used to perform the MD simulation. The binding free energy between the $\mathrm{E}$ dimer proteins of 
DENV was $5,204.22 \mathrm{kcal} / \mathrm{mol}$ at low $\mathrm{pH}$, which is relatively more positive than $3,786.63 \mathrm{kcal} / \mathrm{mol}$ at normal $\mathrm{pH}$. The positive free energy may be caused by destabilization without the $\mathrm{M}$ proteins and bilayer. However, the unfavourable protonation-induced binding between units is similar to our results in which there are increased energies in the low_pH system. Two large differences between the normal and low $\mathrm{pH}$ in DENV were the electrostatic and polar interactions (2,602.79 vs. 26,027.97 kcal/mol and 1,081.75 vs. $-20,942.92$ $\mathrm{kcal} / \mathrm{mol}$, respectively), which also exist in ZIKV (-407.80 vs. 2,257.55 $\mathrm{kcal} / \mathrm{mol}$ and 506.21 vs. $-2,153.03 \mathrm{kcal} / \mathrm{mol}$, respectively). In the low_pH system, protonation increased the repulsive force between the positive charges, whereas weakened interactions led to increased SASAs, causing the polar interactions to increase. The SASAs of the proteins will be discussed in detail in the following sections. Moreover, the weakened interactions also led to the slightly decreased Van der Waals interactions (-182.47 vs. $-133.46 \mathrm{kcal} / \mathrm{mol})$ in our calculations. These interactions show that protonation makes the binding of the E protein not energetically favoured in the low_pH system. The consistency between the DCCM and energetic analyses supports the conclusion that an acidic $\mathrm{pH}$ may affect the integration of the dimer and lower the residue-residue correlations and is critical for the activation of the rearrangement.

\section{The role of the histidine residues}

Histidines are critical in membrane fusion under the histidine switch hypothesis, in which protonation would be the initial trigger. Thus, we focused our attention on the histidine residues of both trajectories to elucidate their key roles in inducing the conformational change (Figs. 5 and 6). Figures 5A and 5B show the side and top views of the alignments of the low_pH (cyan) and neutral_pH (white) states. Figure 6A shows the time evolution for the number of H-bonds in each system. The low_pH system had a lower number of 
H-bonds than the neutral_pH system over time due to the effect of histidine's protonation.

Four obviously distinct H-bond binding states are considered where the histidine residues are located. (1) Figures 5C, 6B and 6C show that in the neutral_pH system, the H-bonds between Glu26 and Arg1025 (an O $\cdots \mathrm{H}-\mathrm{N}$ distance of $2.1 \AA$ in Fig. 5C) closed over time. However, in the low_pH system, the H-bond, Glu26-Hip288, formed at approximately $10 \mathrm{~ns}$ (Fig. 6B). Then, the H-bonds between Glu26 and Arg1025 broke at approximately 20 ns (Fig. 6C). The side chain of Arg1025 rotated away from Glu26 (Fig. 5C). The H-bond formed between Glu26 and both the main chain and the protonated side chain of Hip288 (an $\mathrm{O} \cdots \mathrm{H}-\mathrm{N}$ distance of $1.8 \AA$ and an $\mathrm{O} \cdots \mathrm{H}-\mathrm{N}$ distance of $2.1 \AA$ in Fig. 5C). (2) As shown in Figs. 5D, 6D and 6E, in the neutral_pH system, the

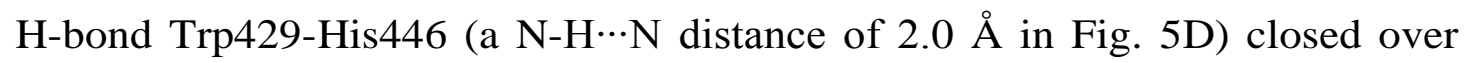
time. However, in the low_pH system, the protonation of His446 directly broke the Trp429-His446 H-bond and formed another H-bond, Asp426-Hip446 (an $\mathrm{O} \cdots \mathrm{H}-\mathrm{N}$ distance of $1.7 \AA$ in Fig. 5D). (3) As shown in Figs. 5E and 6F, in the

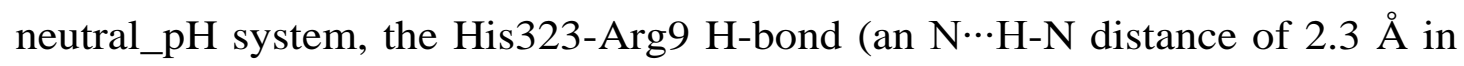
Fig. 5E) closed after approximately 80 ns (Fig. 6F). However, the Hip323-Arg9 H-bond did not form (an $\mathrm{N} \cdots \mathrm{H}-\mathrm{N}$ distance of $6.6 \AA$ in Fig. 5E) over time because of the protonation. (4) As shown in Figs. 5F, 6G and 6H, in the neutral_pH system, the H-bond between Asp98 and Ser512 (with an O $\cdots \mathrm{H}-\mathrm{N}$ distance of $1.6 \AA$ in Fig. 5F) closed over time. The Asp98-His249 H-bond did not form. However, in the low_pH system, the Asp98-Ser512 H-bond broke because of Hip249, which is the protonated histidine residue. The Asp98-Hip249 H-bond (an $\mathrm{O} \cdots \mathrm{H}-\mathrm{N}$ distance of $1.8 \AA$ in Fig. 5F) closed at approximately $25 \mathrm{~ns}$ for the first time and remained steady from 70 to $140 \mathrm{~ns}$ (Fig. 6H). In the meantime, the Asp98-Ser512 H-bond broke at approximately 25 ns. Generally, a H-bond is effective if its occupancy is beyond 30\%[32]. Thus, the protonated Hip249 may break the Asp98-Ser512 H-bond. Figure S4 
shows that the binding modes of the same H-bond sites in another monomer are similar except for site (4).

The four histidine residues that we focused on, His249, His288, His323 and His446, are highly conserved in the E proteins of all flaviviruses, such as DENV, TBEV and ZIKV[45]. Additionally, the residues Arg9, Glu26, Asp98, Asp426, Trp429 and Arg1025, which are involved in forming H-bonds with histidine residues, are also highly conserved[46]. Thus, these histidine residues play an important role in the initiation of the multistep process of membrane fusion. Glu26, His288 and Arg1025 of the H-bond site (1) were located at the interface of the DI of the E protein and the stem region of the M protein (Fig. 1). The protonation of His288 broke the original H-bonds between Glu26 and Arg1025 and led to an inner H-bond in monomer 1, weakening the interaction between the E and M proteins. Asp426, Trp429 and His446 of the H-bond site (2) were located in the stem region of the E protein. The protonation of His446 changed its binding mode and increased the distance of the neighbouring stem helixes. The previous computational studies always focused on the E protein of the flavivirus with the limit of the crystal structures[31,32,34,47]. However, the stem region contributes to a stable intermediate in the late stage of membrane fusion[49]. However, in the early stage, as our MD simulations demonstrate, the binding modes of the H-bonds involving the stem region changed because of the protonation of the histidines. This may have made the integration uncompact (Fig. 3A), with increasing distances for D1 and D2.

Arg9 and His323 of H-bond site (3) were located in the DI and DIII, respectively. The integrity of the DI and DIII is critical for the dimer because the fusion process requires a rearrangement to expose the FL (Fig. 7). The protonation of His323 destabilizes the DI/DIII interface by breaking the interaction with Arg9 and leads to the dissolution of domain interactions. Fig. S5 shows the comparison of the prefusion and postfusion crystal structure of DENV (PDB ID: 1OAN[40] and 1OK8[72], respectively). The relative 
positions of loop1 and loop2 changed after the conformational rearrangement. Thus, the DIII may move apart from the DI in the direction of the black arrow (Fig. 7) to make loop1 cross over loop2 during the early stage of membrane fusion. The destabilization is beneficial to expose the fusion loop to the solvent. It has been confirmed that His323 plays a dominant role as a $\mathrm{pH}$ sensor to drive the initiation of the fusion process in the E proteins of flaviviruses, such as TBEV and DENV[34,44]. To depict the behaviour of the fusion loop, we calculated the PMF for distance D3 vs. D4 (Fig. S6), where D3 is the distance between the COM of the fusion loop in monomer 1 and the COM of DI and DIII in monomer 2, and D4 is the distance between the COM of the fusion loop in monomer 1 and the TM region in monomer 1 . The PMF map clearly shows the low_pH and the neutral_pH states, whereas the neutral_pH state is almost the same as the initial state in the PMF map.

Asp98, Ser512 and His249 of the H-bond site (4) were located at the monomer 1/2 interface. The original H-bonds between Asp98 and Ser512 maintained the integration of the dimer. The protonation of His249 broke the H-bonds and weakened the interaction between the two monomers. However, the relatively protonated Hip754 in another monomer interface did not break the Asp603-Ser7 interaction. The conformational change may not be synchronous among monomers, possibly leading to asymmetric H-bond sites. In the TBEV E protein, His249, along with His288 and His446, three conserved histidine residues (corresponding to His248, His287 and His438 in TBEV), have been ruled out as $\mathrm{pH}$ sensors for the process of membrane fusion by mutational analysis and may be involved in other $\mathrm{pH}$-dependent processes[44]. Thus, the protonation of these histidine residues is probably not crucial for initiation of membrane fusion in the ZIKV E protein. However, the binding mode changes of the H-bonds in our MD simulations show that these histidine residues have a cooperative effect and contribute to weakening the interactions and promoting the fusion process. Besides, we also pay attention to other 
protonable residues such as aspartic acids and glutamic acids. Their contributions that lead to conformational change were not obvious in the two system.

\section{The gap between the monomers}

The weakened residue-residue correlations of the homodimer widen the gap between monomers 1 and 2, which are filled with water molecules. To measure them quantifiably, we counted the number of water molecules in the gap by building a $60 \times 28 \times 26 \AA^{3}$ box. The centre of the box was placed at the centre of mass of residues 1-402 and 506-907. The longest side was positioned parallel to the $\mathrm{x}$-axis. Figure $8 \mathrm{~A}$ shows the time evolution of the number of water molecules in each system. In the low_pH system, there were approximately 500 water molecules in the gap. However, in the neutral_pH system, there were approximately 460 water molecules in the gap. Figures 8B and S4 illustrate the side and top views, respectively, of the measured water boxes of the two representative states. The low_pH state has more water molecules than the neutral_pH state (yellow circles in Fig. 8B), indicating a much looser conformation.

The process of membrane fusion requires structural arrangement; thus, monomer exposure to the solvent is inevitable. We calculated the SASAs of the DI, DII and DIII of the low_pH and neutral_pH systems. In the neutral_pH system, the SASA was $35,823.0 \AA^{2}$, whereas the SASA was $38,041.1 \AA^{2}$ in the low_pH system. The enlarged SASA of the low_pH system was the same as the widened gap and was derived from the weakened interactions between the monomers, which was induced by the protonation of the histidines. The SASAs of the FL of each system were also calculated. The SASAs of the low_pH and neutral_pH systems were $945.5 \AA^{2}$ and $783.9 \AA^{2}$, respectively. The hydrophobic FL was exposed during membrane fusion; thus, the enlarged SASA of the low_pH system is reasonable. 


\section{Conclusions}

To explore the conformational changes at low $\mathrm{pH}$ and investigate the pH-introduced membrane fusion mechanism, we performed two 220-ns MD simulations (20-ns cMD and 200-ns aMD simulations) for ZIKV (E-M) embedded in the POPC membrane for different protonated states of the histidines. The aMD simulations were used for enhancing the conformational space sampling to overcome the time limitation of cMD simulations. The potential of mean force calculation (PMF) was used to study the conformational changes. The acidic environment weakened the H-bond network in both E-protein monomers and in the E-M heterodimer, and it decreases the residue-residue correlations around the contact surface of the $(E-M)_{2}$ homodimers. The highly conserved histidine residues, His249, His288, His323 and His446, play key roles in driving the process of membrane fusion. During the entire MD production, all of the binding energies between (1) the E dimer proteins, (2) the E dimer and $\mathrm{M}$ proteins and (3) the E dimer and the bilayer increased, whereas the solvent accessible surface area (SASA) of FL increased, indicating that the trend for $(\mathrm{E}-\mathrm{M})_{2}$ homodimers depolymerization was triggered by the protonation of the histidines.

The results and analyses of our MD simulations may provide insights into the molecular mechanism of ZIKV infection. A low $\mathrm{pH}$ environment is necessary for activation of Class II fusion proteins, such as our targeted ZIKV E protein. Protonation of highly conserved histidine residues weakens the interactions between each domain and drives conformational changes. This mechanistic study should facilitate finding a target for the future design of potent ZIKV inhibitors and may provide assistance in finding treatments for ZIKV-caused epidemic diseases.

\section{Acknowledgments}


This study was supported by the National Science Foundation of China (No .31070640) and the Ph.D. Candidate Research Innovation Fund of Nankai University.

\section{References}

[1] J.M. Medlock, K.M. Hansford, F. Schaffner, V. Versteirt, G. Hendrickx, H. Zeller, W. Van Bortel, A Review of the Invasive Mosquitoes in Europe: Ecology, Public Health Risks, and Control Options, Vector Borne Zoonotic Dis. 12 (2012) 435-447. doi:10.1089/vbz.2011.0814.

[2] M. McCarthy, Vectors for Zika virus may spread further than was previously thought, CDC reports, Br. Med. J. 353 (2016). doi:10.1136/bmj.i1914.

[3] L.C. Rodrigues, Microcephaly and Zika virus infection, Lancet. 387 (2016) 2070-2072. doi:10.1016/S0140-6736(16)00742-X.

[4] L. Schuler-Faccini, E.M. Ribeiro, I.M.L. Feitosa, D.D.G. Horovitz, D.P. Cavalcanti, A. Pessoa, M.J.R. Doriqui, J.I. Neri, J.M. de Pina Neto, H.Y.C. Wanderley, M. Cernach, A.S. El-Husny, M.V.S. Pone, C.L.C. Serao, M.T. V Sanseverino, E. Brazilian Med Genet Soc Zika, Possible Association Between Zika Virus Infection and Microcephaly - Brazil, 2015, Morb. Mortal. Wkly. Rep. 65 (2016) 59-62.

[5] D.W. Smith, J. Mackenzie, Zika virus and Guillain-Barre syndrome: another viral cause to add to the list, Lancet. 387 (2016) 1486-1488. doi:10.1016/s0140-6736(16)00564-x.

[6] C. Zanluca, V.C.A. de Melo, A.L.P. Mosimann, G.I.V. dos Santos, C.N.D. dos Santos, K. Luz, First report of autochthonous transmission of Zika virus in Brazil, Mem. Inst. Oswaldo Cruz. 110 (2015) 569-572. doi:10.1590/0074-02760150192.

[7] W.H. Organization, Zika strategic response framework and joint operations plan, January-June 2016, (2016).

[8] E. Petersen, M.E. Wilson, S. Touch, B. McCloskey, P. Mwaba, M. Bates, O. Dar, F. Mattes, M. Kidd, G. Ippolito, E.I. Azhar, A. Zumla, Rapid Spread of Zika Virus in The Americas Implications for Public Health Preparedness for Mass Gatherings at the 2016 Brazil Olympic Games, Int. J. Infect. Dis. 44 (2016) 11-15. doi:10.1016/j.ijid.2016.02.001.

[9] J.S. Mackenzie, D.T. Williams, The Zoonotic Flaviviruses of Southern, South-Eastern and Eastern Asia, and Australasia: The Potential for Emergent Viruses, Zoonoses Public Health. 56 (2009) 338-356. doi:10.1111/j.1863-2378.2008.01208.x.

[10] D. Musso, D.J. Gubler, Zika Virus, Clin. Microbiol. Rev. 29 (2016) 487-524. doi:10.1128/cmr.00072-15.

[11] G. Kuno, G.J.J. Chang, Full-length sequencing and genomic characterization of Bagaza, Kedougou, and Zika viruses, Arch. Virol. 152 (2007) 687-696. doi:10.1007/s00705-006-0903-z.

[12] E.B. Hayes, Zika Virus Outside Africa, Emerg. Infect. Dis. 15 (2009) 1347-1350. doi:10.3201/eid1509.090442.

[13] R.J. Kuhn, W. Zhang, M.G. Rossmann, S. V Pletnev, J. Corver, E. Lenches, C.T. Jones, S. Mukhopadhyay, P.R. Chipman, E.G. Strauss, Structure of dengue virus: implications for flavivirus organization, maturation, and fusion, Cell. 108 (2002) 717-725.

[14] L. Li, S.-M. Lok, I.-M. Yu, Y. Zhang, R.J. Kuhn, J. Chen, M.G. Rossmann, The flavivirus 
precursor membrane-envelope protein complex: structure and maturation, Science (80-. ). 319 (2008) 1830-1834.

[15] O. Faye, C.C.M. Freire, A. Iamarino, O. Faye, J.V.C. de Oliveira, M. Diallo, P.M.A. Zanotto, A.A. Sall, Molecular Evolution of Zika Virus during Its Emergence in the 20th Century, PLoS Negl. Trop. Dis. 8 (2014). doi:e263610.1371/journal.pntd.0002636.

[16] Y. Zhang, J. Corver, P.R. Chipman, W. Zhang, S. V Pletnev, D. Sedlak, T.S. Baker, J.H. Strauss, R.J. Kuhn, M.G. Rossmann, Structures of immature flavivirus particles, EMBO J. 22 (2003) 2604-2613. doi:10.1093/emboj/cdg270.

[17] T. Reddy, M.S.P. Sansom, The role of the membrane in the structure and biophysical robustness of the dengue virion envelope, Structure. 24 (2016) 375-382.

[18] T.S. Jardetzky, R. a Lamb, Virology: a class act., Nature. 427 (2004) 307-308. doi:10.1038/427307a.

[19] M. Kielian, Class II virus membrane fusion proteins, Virology. 344 (2006) 38-47. doi:10.1016/j.virol.2005.09.036.

[20] K. Stiasny, Flavivirus membrane fusion, J. Gen. Virol. 87 (2006) 2755-2766. doi:10.1099/vir.0.82210-0.

[21] B. Kaufmann, P.R. Chipman, H.A. Holdaway, S. Johnson, D.H. Fremont, R.J. Kuhn, M.S. Diamond, M.G. Rossmann, Capturing a flavivirus pre-fusion intermediate, PLoS Pathog. 5 (2009) 4-8. doi:10.1371/journal.ppat.1000672.

[22] S.L. Allison, J. Schalich, K. Stiasny, C.W. Mandl, F.X. Heinz, Mutational evidence for an internal fusion peptide in flavivirus envelope protein E, J. Virol. 75 (2001) 4268-4275. doi:10.1128/jvi.75.9.4268-4275.2001.

[23] S.C. Harrison, Viral membrane fusion, Nat. Struct. Mol. Biol. 15 (2008) 690-698. doi:10.1038/nsmb.1456.

[24] L.J. Earp, S.E. Delos, H.E. Park, J.M. White, The many mechanisms of viral membrane fusion proteins., Curr. Top. Microbiol. Immunol. 285 (2005) 25-66. doi:10.1007/3-540-26764-6_2.

[25] S. Mukhopadhyay, R.J. Kuhn, M.G. Rossmann, A structural perspective of the flavivirus life cycle., Nat. Rev. Microbiol. 3 (2005) 13-22. doi:10.1038/nrmicro1067.

[26] I. Yu, W. Zhang, H.A. Holdaway, L. Li, V.A. Kostyuchenko, P.R. Chipman, R.J. Kuhn, M.G. Rossmann, J. Chen, Structure of the Immature Dengue Virus at Low pH Primes Proteolytic Maturation, Science (80-. ). 319 (2008) 1834-1837. doi:10.1126/science.1153264.

[27] D.M. Eckert, P.S. Kim, Mechanisms of viral membrane fusion and its inhibition, Annu. Rev. Biochem. 70 (2001) 777-810.

[28] T.C. Pierson, M.S. Diamond, Degrees of maturity: the complex structure and biology of flaviviruses, Curr. Opin. Virol. 2 (2012) 168-175. doi:10.1016/j.coviro.2012.02.011.

[29] K. Stiasny, R. Fritz, K. Pangerl, F.X. Heinz, Molecular mechanisms of flavivirus membrane fusion, Amino Acids. 41 (2011) 1159-1163. doi:10.1007/s00726-009-0370-4.

[30] M. Liao, M. Kielian, Domain III from class II fusion proteins functions as a dominant-negative inhibitor of virus membrane fusion, J. Cell Biol. 171 (2005) 111-120. doi:10.1083/jcb.200507075.

[31] M.K. Prakash, A. Barducci, M. Parrinello, Probing the mechanism of pH-induced large-scale conformational changes in dengue virus envelope protein using atomistic simulations, Biophys. J. 99 (2010) 588-594.

[32] K.D. Dubey, A.K. Chaubey, R.P. Ojha, Role of pH on dimeric interactions for DENV envelope 
protein: An insight from molecular dynamics study, Biochim. Biophys. Acta - Proteins Proteomics. 1814 (2011) 1796-1801. doi:10.1016/j.bbapap.2011.09.012.

[33] K. Pangerl, F.X. Heinz, K. Stiasny, Mutational Analysis of the Zippering Reaction during Flavivirus Membrane Fusion, J. Virol. 85 (2011) 8495-8501. doi:10.1128/jvi.05129-11.

[34] C.A. Fuzo, Le. Degreve, The $\mathrm{pH}$ dependence of flavivirus envelope protein structure: insights from molecular dynamics simulations, J. Biomol. Struct. Dyn. 32 (2014) 1563-1574. doi:10.1080/07391102.2013.827132.

[35] D.M. Rogers, M.S. Kent, S.B. Rempe, Molecular basis of endosomal-membrane association for the dengue virus envelope protein, Biochim. Biophys. Acta-Biomembranes. 1848 (2015) 1041-1052. doi:10.1016/j.bbamem.2014.12.018.

[36] T. Reddy, M.S.P. Sansom, Computational Virology: from the Inside Out, Biochim. Biophys. Acta - Biomembr. (2016). doi:10.1016/j.bbamem.2016.02.007.

[37] Y.S. Mendes, N.S. Alves, T.L.F. Souza, I.P. Sousa, M.L. Bianconi, R.C. Bernardi, P.G. Pascutti, J.L. Silva, A.M.O. Gomes, A.C. Oliveira, The Structural Dynamics of the Flavivirus Fusion Peptide-Membrane Interaction, PLoS One. 7 (2012). doi:10.1371/journal.pone.0047596.

[38] S. Bressanelli, K. Stiasny, S.L. Allison, E. a Stura, S. Duquerroy, J. Lescar, F.X. Heinz, F. a Rey, Structure of a flavivirus envelope glycoprotein in its low-pH-induced membrane fusion conformation, EMBO J. 23 (2004) 728-738. doi:10.1038/sj.emboj.7600064.

[39] F.A. Rey, F. Heinz, X., C. Mandl, C. Kunz, S.C. Harrison, The envelope glycoprotein from tick-borne encephalitis virus at $2 \AA$ resolution, Nature. 375 (1995) 291-298. doi:10.1038/375291a0.

[40] Y. Modis, S. Ogata, D. Clements, S.C. Harrison, A ligand-binding pocket in the dengue virus envelope glycoprotein, Proc. Natl. Acad. Sci. U. S. A. 100 (2003) 6986-6991.

[41] R. Kanai, K. Kar, K. Anthony, L.H. Gould, M. Ledizet, E. Fikrig, W.A. Marasco, R.A. Koski, Y. Modis, Crystal structure of West Nile virus envelope glycoprotein reveals viral surface epitopes, J. Virol. 80 (2006) 11000-11008. doi:10.1128/jvi.01735-06.

[42] V.C. Luca, J. AbiMansour, C.A. Nelson, D.H. Fremont, Crystal Structure of the Japanese Encephalitis Virus Envelope Protein, J. Virol. 86 (2012) 2337-2346. doi:10.1128/jvi.06072-11.

[43] T. Kampmann, D.S. Mueller, A.E. Mark, P.R. Young, B. Kobe, The Role of Histidine Residues in Low-pH-Mediated Viral Membrane Fusion, Structure. 14 (2006) 1481-1487. doi:10.1016/j.str.2006.07.011.

[44] R. Fritz, K. Stiasny, F.X. Heinz, Identification of specific histidines as $\mathrm{pH}$ sensors in flavivirus membrane fusion, J. Cell Biol. 183 (2008) 353-361. doi:10.1083/jcb.200806081.

[45] V.A. Kostyuchenko, E.X.Y. Lim, S. Zhang, G. Fibriansah, T.-S. Ng, J.S.G. Ooi, J. Shi, S.-M. Lok, Structure of the thermally stable Zika virus, Nature. 533 (2016) 425-428. doi:10.1038/nature17994.

[46] D. Sirohi, Z. Chen, L. Sun, T. Klose, T.C. Pierson, M.G. Rossmann, R.J. Kuhn, The 3.8 A resolution cryo-EM structure of Zika virus, Science (80-. ). 352 (2016) 467-470. doi:10.1126/science.aaf5316.

[47] D.S. Mueller, T. Kampmann, R. Yennamalli, P.R. Young, B. Kobe, A.E. Mark, Histidine protonation and the activation of viral fusion proteins., Biochem. Soc. Trans. 36 (2008) 43-5. doi:10.1042/BST0360043.

[48] D. Hamelberg, J. Mongan, J.A. McCammon, Accelerated molecular dynamics: A promising 
and efficient simulation method for biomolecules, J. Chem. Phys. 120 (2004) 11919-11929. doi:10.1063/1.1755656.

[49] D.E. Klein, J.L. Choi, S.C. Harrison, Structure of a Dengue Virus Envelope Protein Late-Stage Fusion Intermediate, J. Virol. 87 (2013) 2287-2293. doi:10.1128/jvi.02957-12.

[50] R. Anandakrishnan, B. Aguilar, A. V. Onufriev, H++ 3.0: Automating pK prediction and the preparation of biomolecular structures for atomistic molecular modeling and simulations, Nucleic Acids Res. 40 (2012) 537-541. doi:10.1093/nar/gks375.

[51] W. Humphrey, A. Dalke, K. Schulten, VMD: Visual molecular dynamics, J. Mol. Graph. 14 (1996) 33-38. doi:http://dx.doi.org/10.1016/0263-7855(96)00018-5.

[52] W.L. Jorgensen, J. Chandrasekhar, J.D. Madura, R.W. Impey, M.L. Klein, Comparison of simple potential functions for simulating liquid water, J. Chem. Phys. 79 (1983) 926-935.

[53] D.A. Case, J.T. Berryman, R.M. Betz, D.S. Cerutti, T.E. Cheatham, T.A. Darden, R.E. Duke, T.J. Giese, H. Gohlke, A.W. Goetz, N. Homeyer, S. Izadi, P. Janowski, J. Kaus, A. Kovalenko, T.S. Lee, S. LeGrand, P. Li, T. Luchko, R. Luo, B. Madej, K.M. Merz, G. Monard, P. Needham, H. Nguyen, H.T. Nguyen, I. Omelyan, A. Onufriev, D.R. Roe, A. Roitberg, R. Salomon Ferrer, C.L. Simmerling, W. Smith, J. Swails, R.C. Walker, J. Wang, R.M. Wolf, X. Wu, D.M. York, P.A. Kollman, AMBER, 14. (2015).

[54] J.A. Maier, C. Martinez, K. Kasavajhala, L. Wickstrom, K.E. Hauser, C. Simmerling, ff14SB: Improving the Accuracy of Protein Side Chain and Backbone Parameters from ff99SB, J. Chem. Theory Comput. 11 (2015) 3696-3713. doi:10.1021/acs.jctc.5b00255.

[55] C.J. Dickson, B.D. Madej, Å.A. Skjevik, R.M. Betz, K. Teigen, I.R. Gould, R.C. Walker, Lipid14: The Amber Lipid Force Field, J. Chem. Theory Comput. 10 (2014) 865-879. doi:10.1021/ct4010307.

[56] K.N. Kirschner, A.B. Yongye, S.M. Tschampel, J. González - Outeiriño, C.R. Daniels, B.L. Foley, R.J. Woods, GLYCAM06: a generalizable biomolecular force field. Carbohydrates, J. Comput. Chem. 29 (2008) 622-655.

[57] R. Fletcher, M.J.D. Powell, A Rapidly Convergent Descent Method for Minimization, Comput. J. 6 (1963) 163-168. doi:10.1093/comjnl/6.2.163.

[58] R. Fletcher, C.M. Reeves, Function minimization by conjugate gradients, Comput. J. 7 (1964) 149-154. doi:10.1093/comjnl/7.2.149.

[59] R.W. Pastor, B.R. Brooks, A. Szabo, An analysis of the accuracy of Langevin and molecular dynamics algorithms, Mol. Phys. 65 (1988) 1409-1419. doi:10.1080/00268978800101881.

[60] T. Darden, D. York, L. Pedersen, Particle mesh Ewald: An $N \cdot \log (\mathrm{N})$ method for Ewald sums in large systems, J. Chem. Phys. 98 (1993) 10089. doi:10.1063/1.464397.

[61] J.-P. Ryckaert, G. Ciccotti, H.J.. Berendsen, Numerical integration of the cartesian equations of motion of a system with constraints: molecular dynamics of n-alkanes, J. Comput. Phys. 23 (1977) 327-341. doi:10.1016/0021-9991(77)90098-5.

[62] I.G. Tikhonova, B. Selvam, A. Ivetac, J. Wereszczynski, J.A. McCammon, Simulations of Biased Agonists in the $\beta 2$ Adrenergic Receptor with Accelerated Molecular Dynamics, Biochemistry. 52 (2013) 5593-5603. doi:10.1021/bi400499n.

[63] D.R. Roe, T.E. Cheatham III, PTRAJ and CPPTRAJ: software for processing and analysis of molecular dynamics trajectory data, J. Chem. Theory Comput. 9 (2013) 3084-3095.

[64] P. Kollman, Free-Energy Calculations - Applications to Chemical and Biochemical Phenomena, Chem. Rev. 93 (1993) 2395-2417. doi:10.1021/cr00023a004. 
[65] L.T. Chong, Y. Duan, L. Wang, I. Massova, P.A. Kollman, Molecular dynamics and free-energy calculations applied to affinity maturation in antibody 48G7, Proc. Natl. Acad. Sci. U. S. A. 96 (1999) 14330-14335. doi:10.1073/pnas.96.25.14330.

[66] P.A. Kollman, I. Massova, C. Reyes, B. Kuhn, S.H. Huo, L. Chong, M. Lee, T. Lee, Y. Duan, W. Wang, O. Donini, P. Cieplak, J. Srinivasan, D.A. Case, T.E. Cheatham, Calculating structures and free energies of complex molecules: Combining molecular mechanics and continuum models, Acc. Chem. Res. 33 (2000) 889-897. doi:10.1021/ar000033j.

[67] B. Roux, The calculation of the potential of mean force using computer simulations, Comput. Phys. Commun. 91 (1995) 275-282.

[68] A. Sethi, J. Eargle, A.A. Black, Z. Luthey-Schulten, Dynamical networks in tRNA:protein complexes., Proc. Natl. Acad. Sci. U. S. A. 106 (2009) 6620-5. doi:10.1073/pnas.0810961106.

[69] N.M. Glykos, Software news and updates carma: A molecular dynamics analysis program, J. Comput. Chem. 27 (2006) 1765-1768.

[70] S. Zhang, V.A. Kostyuchenko, T.-S. Ng, X.-N. Lim, J.S.G. Ooi, S. Lambert, T.Y. Tan, D.G. Widman, J. Shi, R.S. Baric, S.-M. Lok, Neutralization mechanism of a highly potent antibody against Zika virus., Nat. Commun. 7 (2016) 13679. doi:10.1038/ncomms13679.

[71] T. Reddy, M.S.P. Sansom, The Role of the Membrane in the Structure and Biophysical Robustness of the Dengue Virion Envelope, Structure. 24 (2016) 375-382. doi:10.1016/j.str.2015.12.011.

[72] Y. Modis, S. Ogata, D. Clements, S.C. Harrison, Structure of the dengue virus envelope protein after membrane fusion., Nature. 427 (2004) 313-319. doi:10.1038/nature02165. 


\section{Figures}

Fig. 1. Structure of the ZIKV E protein. Colour codes of the domains with residue numbers are shown.

Fig. 2. Illustrations of the curvature of bilayer of (A) the low_pH system and (B) the neutral_pH system. TM domains, remainder of protein and lipid are in orange, blue and black/grey, respectively. The snapshot is the last frame of each trajectory. Time evolutions of bilayer thickness proximal (black) and distal (blue) to TM domains of (C) the Low_pH system and (D) the Neutral_pH system.

Fig. 3. (A) Definitions of distances D1 and D2. The COMs of the domains are depicted by spheres. (B) PMF calculated for distance D1 vs. D2. (C) Time evolution of distance D1 for each system. (D) Time evolution of distance D2 for each system.

Fig. 4. (A) The DCCM for the low_pH and neutral_pH systems. The colour scale is shown on the right, which is from red (highly positive correlations) to blue (highly negative correlations). (B) The alignment of the initial (green), low_pH (cyan) and neutral_pH (white) states is based on DI and DIII.

Fig. 5. Comparison of the neutral_pH (white) and low_pH (cyan) states. (A) Top view. (B) Side view. (C-F) More details are shown in the distinct $\mathrm{H}$-bond binding states in which the histidine residues are located. The residues involved in the specific interactions are labelled and depicted as sticks.

Fig. 6. Time evolutions and distributions of (A) the number of H-bonds, (B) the $\mathrm{O} \cdots \mathrm{H}-\mathrm{N}$ distance between Glu26 and His(Hip)288, (C) the $\mathrm{O} \cdots \mathrm{H}-\mathrm{N}$ distance between Glu26 and Arg1025, (D) the N-H*NN distance between Thr429 and His(Hip)446, (E) the $\mathrm{O} \cdots \mathrm{H}-\mathrm{N}$ distance between Glu429 and His(Hip)446, (F) the N $\cdots \mathrm{H}-\mathrm{N}$ distance between of His(Hip)323 and $\operatorname{Arg} 9,(\mathrm{G})$ the $\mathrm{O} \cdots \mathrm{H}-\mathrm{O}(\mathrm{N})$ distance between Asp98 and Ser512, and $(\mathrm{H})$ the $\mathrm{O} \cdots \mathrm{H}-\mathrm{N}$ distance between Asp98 and His (Hip) 249.

Fig. 7. The broken interaction of His323-Arg9 destabilizes the DI/DIII interface and exposes the fusion loop (orange).

Fig. 8. (A) The time evolutions of the water molecules in the defined box of each system. (B) Illustrations of the differences in the number of water molecules between 
the low_pH (cyan) and neutral_pH (white) states. The water molecules are depicted as a mesh. The differences are labelled by yellow circles.

Table 1 Binding free energies of E-E, E-M and E-P. All free energy contributions are in $\mathrm{kcal} / \mathrm{mol}$.

\begin{tabular}{|c|c|c|c|c|c|}
\hline \multirow{2}{*}{\multicolumn{2}{|c|}{ Contributions }} & \multicolumn{2}{|c|}{ Low_pH } & \multicolumn{2}{|c|}{ Neutral_pH } \\
\hline & & Average & Std. Dev. & Average & Std. Dev. \\
\hline \multirow{7}{*}{ E-E } & $\Delta \mathrm{E}_{\mathrm{vdw}}$ & -133.46 & 6.87 & -182.47 & 7.16 \\
\hline & $\Delta \mathrm{E}_{\text {ele }}$ & 2257.55 & 62.08 & -407.80 & 46.88 \\
\hline & $\Delta \mathrm{E}_{\mathrm{pol}}$ & -2153.03 & 59.60 & 506.21 & 38.67 \\
\hline & $\Delta \mathrm{E}_{\mathrm{npol}}$ & -15.03 & 0.84 & -19.13 & 0.50 \\
\hline & $\Delta \mathrm{G}_{\mathrm{gas}}$ & 2124.09 & 60.32 & -590.27 & 45.79 \\
\hline & $\Delta \mathrm{G}_{\mathrm{solv}}$ & -2168.05 & 59.74 & 487.08 & 38.47 \\
\hline & $\Delta \mathrm{G}_{\mathrm{tot}}$ & -43.97 & 10.46 & -103.19 & 12.51 \\
\hline \multirow{7}{*}{ E-M } & $\Delta \mathrm{E}_{\mathrm{vdw}}$ & -402.82 & 11.83 & -493.08 & 20.59 \\
\hline & $\Delta \mathrm{E}_{\text {ele }}$ & 3432.18 & 79.09 & -1096.26 & 77.13 \\
\hline & $\Delta \mathrm{E}_{\mathrm{pol}}$ & -3226.82 & 73.85 & 1300.77 & 70.53 \\
\hline & $\Delta \mathrm{E}_{\mathrm{npol}}$ & -48.55 & 0.98 & -55.64 & 0.84 \\
\hline & $\Delta \mathrm{G}_{\mathrm{gas}}$ & 3029.36 & 80.92 & -1589.34 & 75.95 \\
\hline & $\Delta \mathrm{G}_{\mathrm{solv}}$ & -3275.36 & 73.68 & 1245.13 & 70.31 \\
\hline & $\Delta \mathrm{G}_{\mathrm{tot}}$ & -246.00 & 21.37 & -344.21 & 19.19 \\
\hline \multirow{7}{*}{ E-P } & $\Delta \mathrm{E}_{\mathrm{vdw}}$ & -542.99 & 37.08 & -643.41 & 42.80 \\
\hline & $\Delta \mathrm{E}_{\text {ele }}$ & -1739.28 & 216.03 & -1265.52 & 129.93 \\
\hline & $\Delta \mathrm{E}_{\mathrm{pol}}$ & 2163.28 & 201.20 & 1735.74 & 130.62 \\
\hline & $\Delta \mathrm{E}_{\mathrm{npol}}$ & -110.26 & 2.07 & -118.85 & 2.91 \\
\hline & $\Delta \mathrm{G}_{\mathrm{gas}}$ & -2282.27 & 220.00 & -1908.94 & 153.28 \\
\hline & $\Delta \mathrm{G}_{\mathrm{solv}}$ & 2053.02 & 200.23 & 1616.89 & 128.80 \\
\hline & $\Delta \mathrm{G}_{\mathrm{tot}}$ & -229.25 & 45.24 & -292.04 & 45.72 \\
\hline
\end{tabular}


Monomer 1

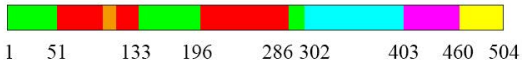

Monomer 2

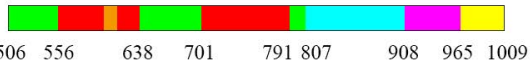

Monomer 1

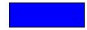

$1011 \quad 1085$

Monomer 2

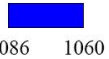

$\square$ Domain I

Domain II

$\square$ Domain III

$\square$ Stem Region $\square$ TM Region $\quad \square$ Fusion Loop

M Protein

\section{Fusion Loop}

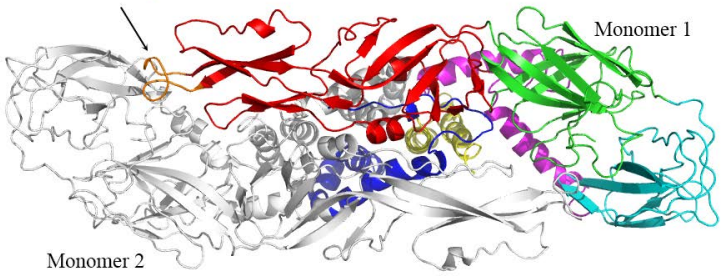


A

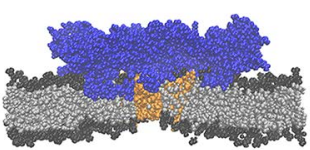

C

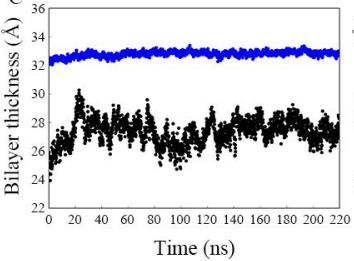

B

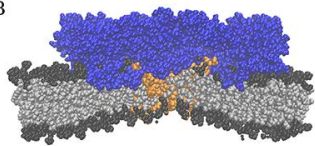

D

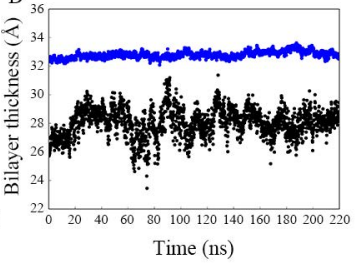


A
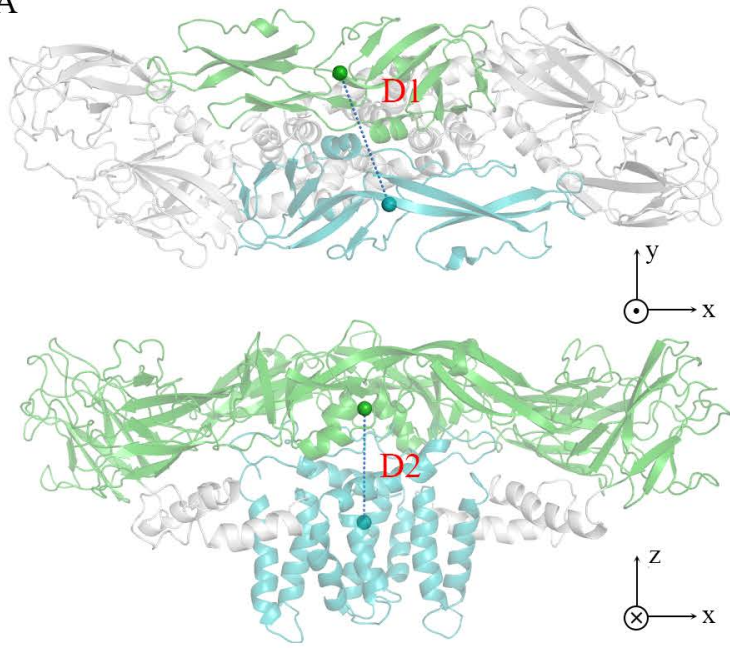

C

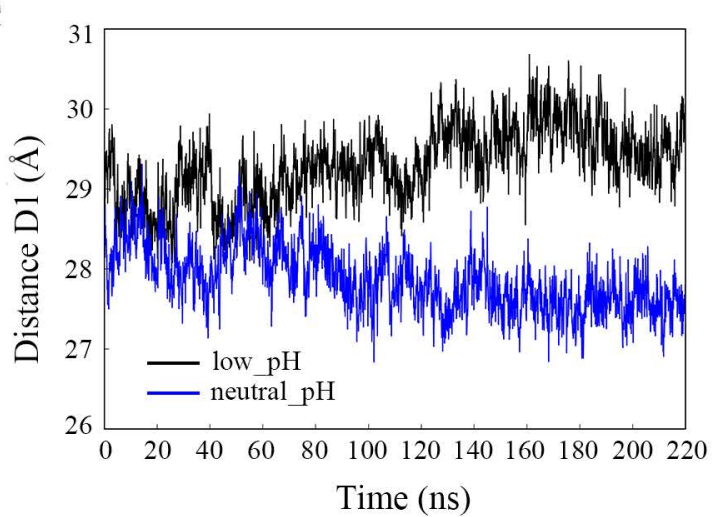

B

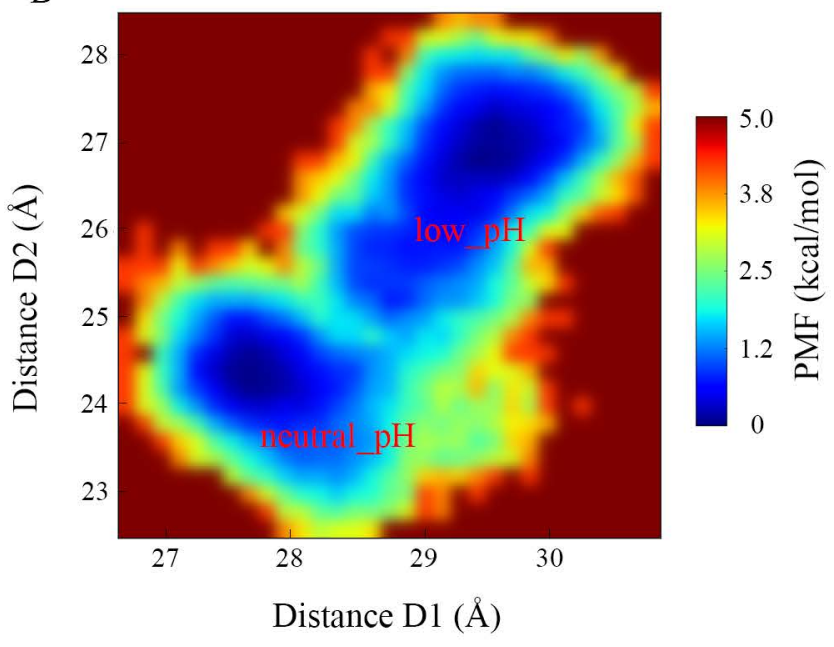

D

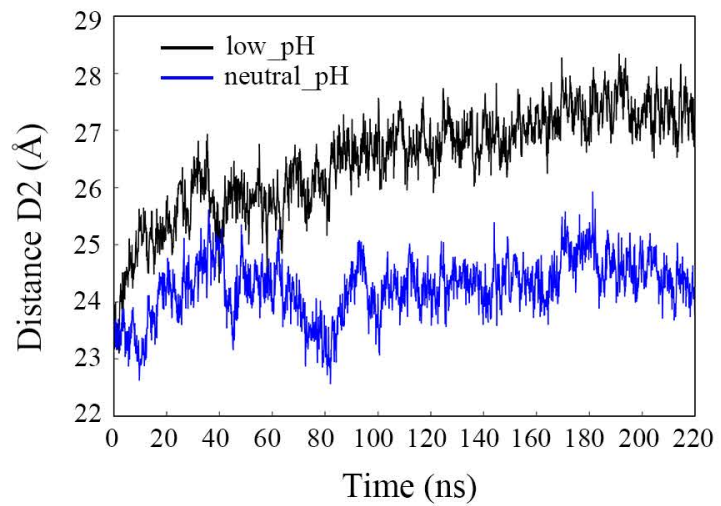


C

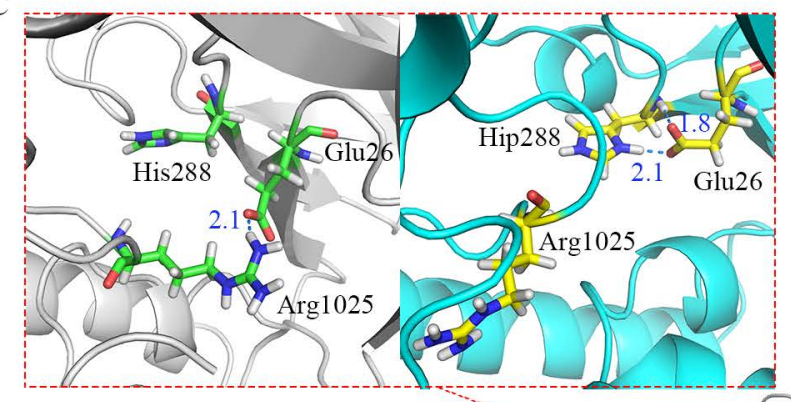

D

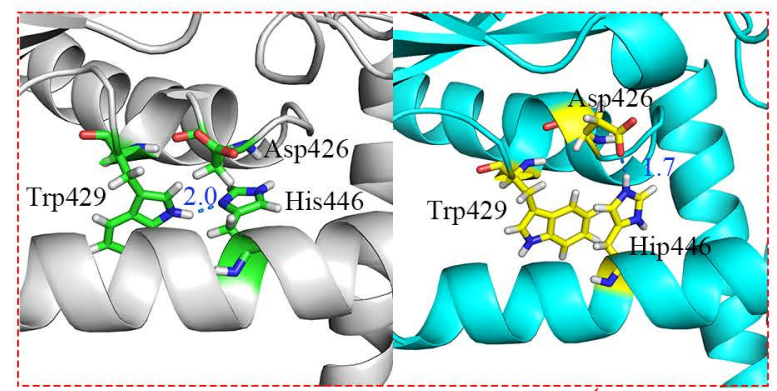

side view

\section{A}

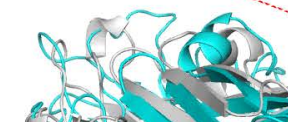

So

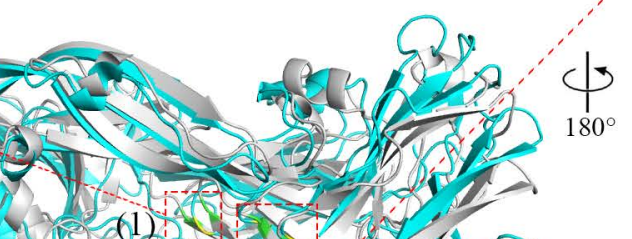

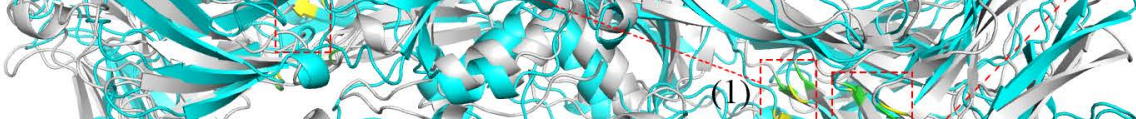

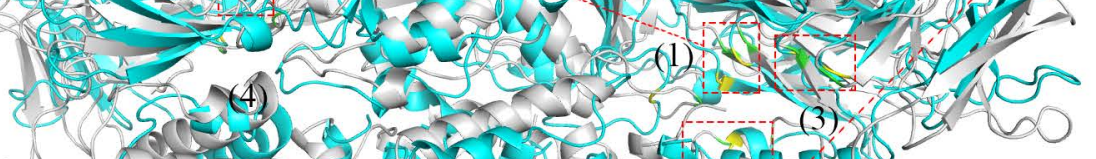

2.

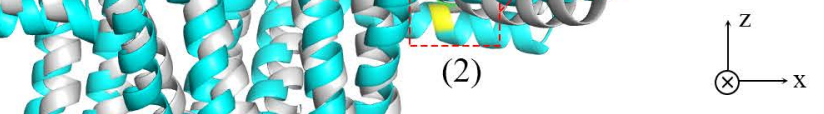

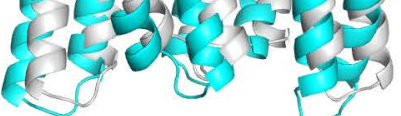

B

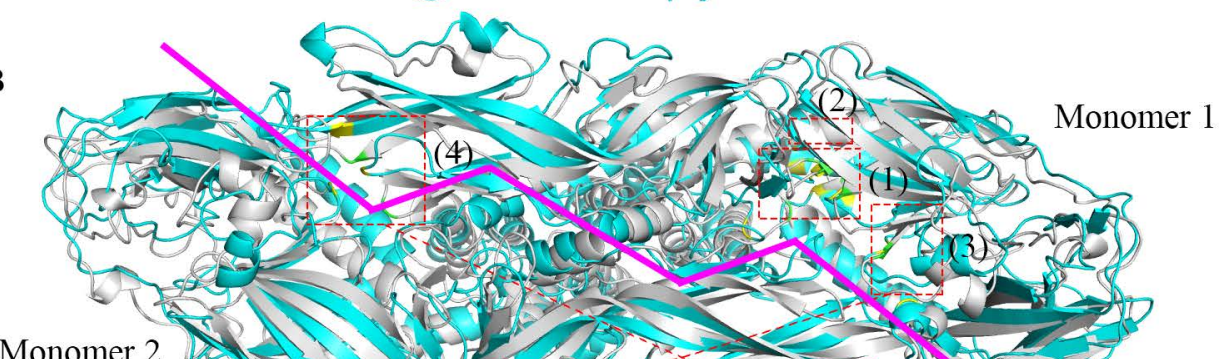

top view

E

Monomer 2 selsents

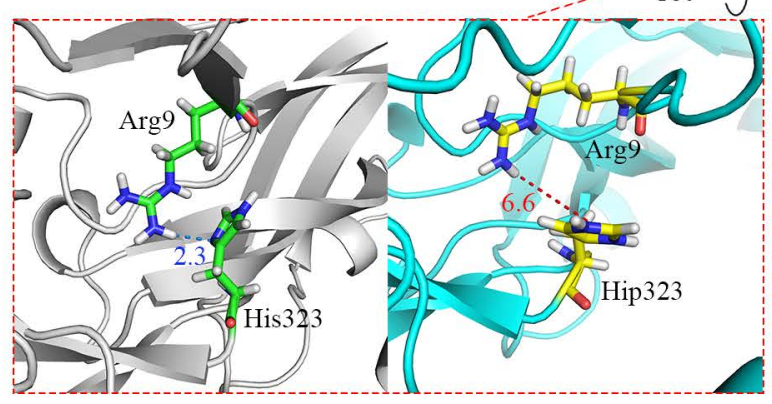

F

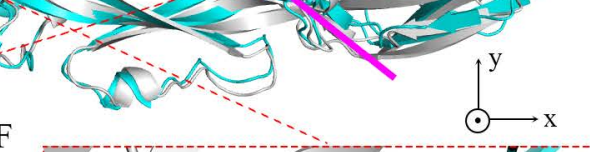

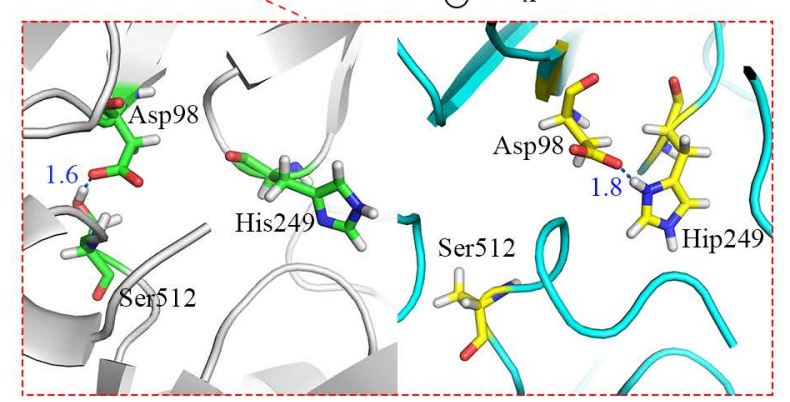



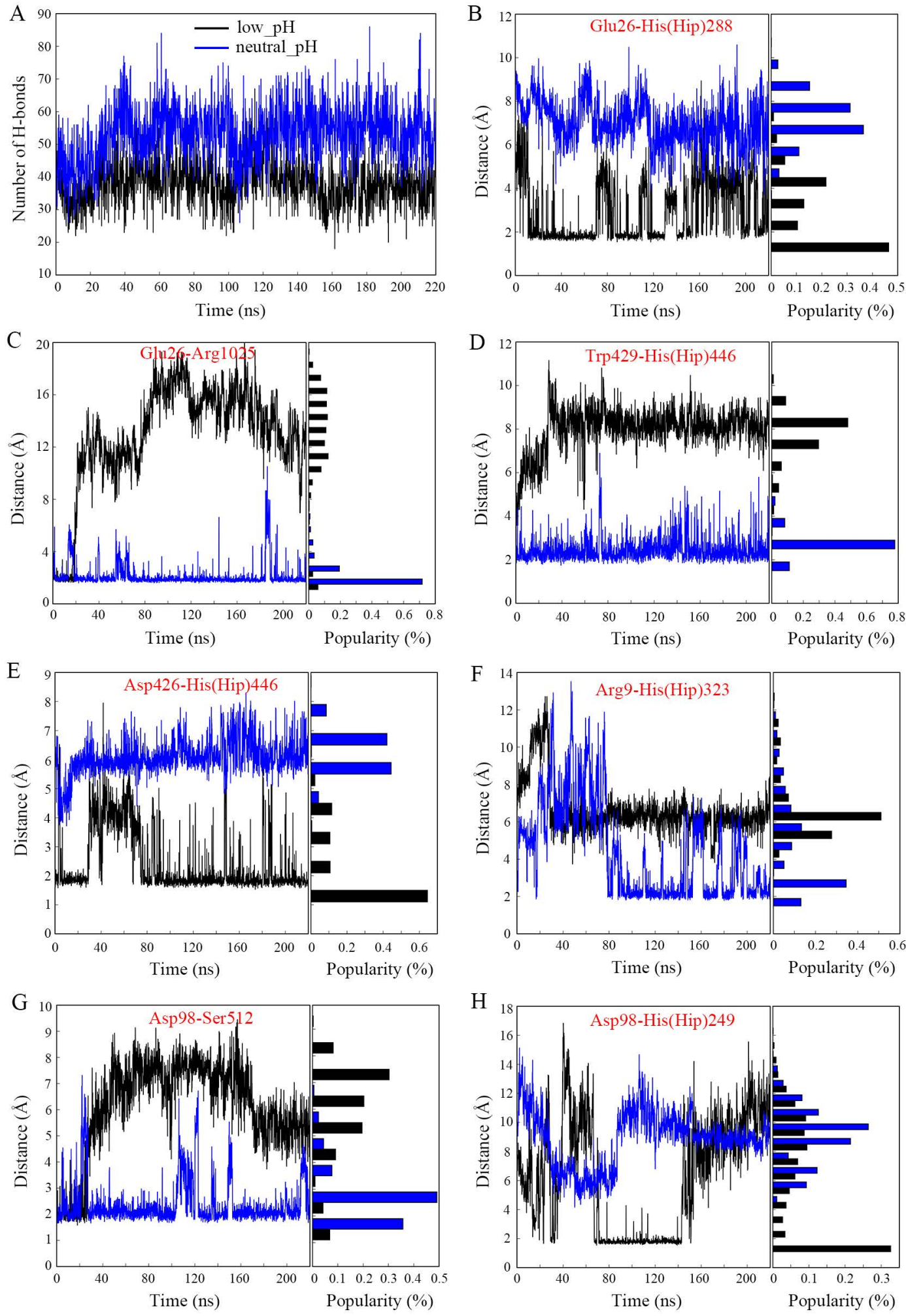


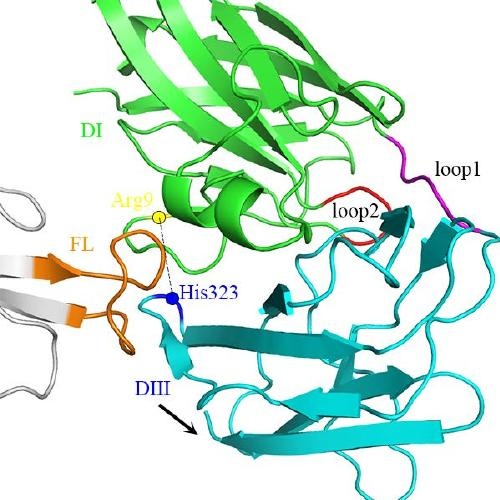




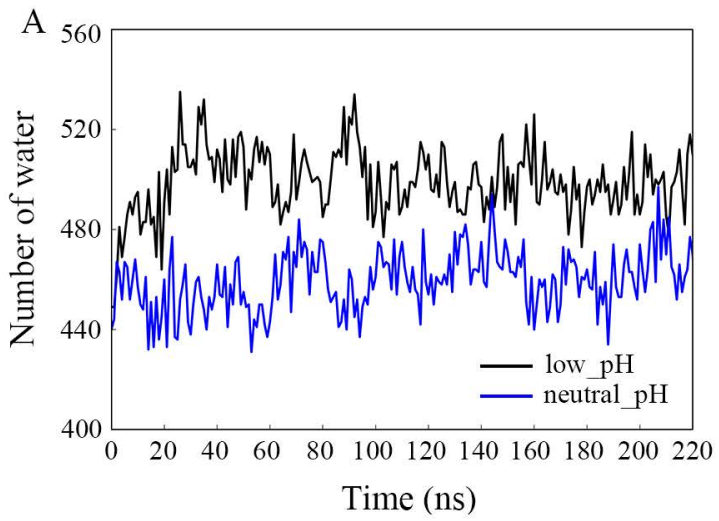

B
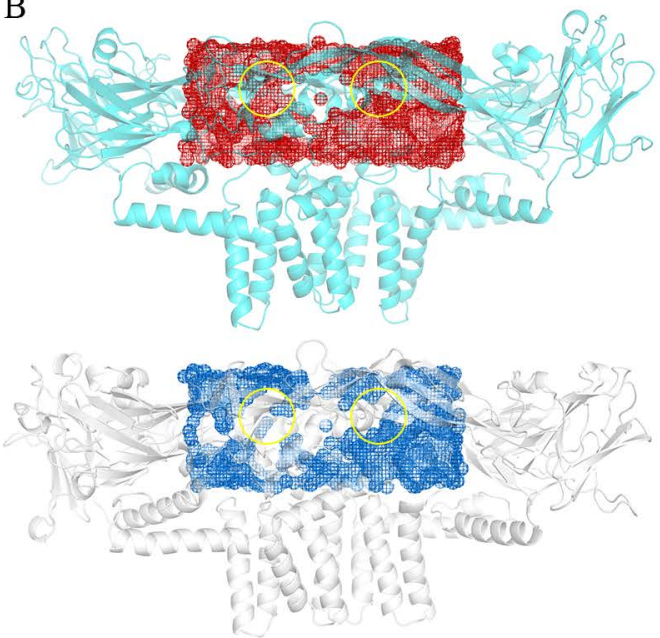